\title{
Affine Killing vector fields on homogeneous surfaces with torsion
}

\author{
D D'Ascanio', P B Gilkey ${ }^{2}$ and P Pisani ${ }^{1,3}$ (B) \\ ${ }^{1}$ Instituto de Física La Plata, CONICET and Universidad Nacional de La Plata, \\ CC 67 (1900) La Plata, Argentina \\ 2 Mathematics Department, University of Oregon, Eugene, OR 97403, \\ United States of America \\ E-mail: dascanio@ fisica.unlp.edu.ar, gilkey@uoregon.edu \\ and pisani@fisica.unlp.edu.ar
}

Received 30 March 2019, revised 23 May 2019

Accepted for publication 6 June 2019

Published 25 June 2019

\begin{abstract}
Many extensions of general relativity are based on considering metric and affine structures as independent properties of spacetime. This leads to the possibility of introducing torsion as an independent degree of freedom. In this article we examine the effects of torsion on the affine Killing vectors of twodimensional manifolds. We give a complete description of the Lie algebras of affine Killing vector fields on homogeneous surfaces. This can be used in the search of non-metrizable surfaces of interest.
\end{abstract}

Keywords: affine connection, affine Killing vector fields, torsion, non-Riemannian surfaces, modified gravity

\section{Introduction}

General relativity is at present the most successful description of the gravitational interaction. However, many open questions settled by the present status of astrophysical observations motivate the search for modified formulations of this theory. In addition, black hole physics and early universe models require a framework compatible with quantum mechanics. For these reasons, general relativity in its present form is not considered as an ultimate description of gravity and different generalizations are currently under study.

One approach to this reformulation is based on a reexamination of the canonical degrees of freedom of the theory. Constructing an invariant action requires a metric and an affine connection, both describing different geometric properties of spacetime. In standard general relativity, only the metric is a fundamental field whereas the affine structure is given by the

${ }^{3}$ Author to whom any correspondence should be addressed. 
Levi-Civita connection. However, from the mathematical point of view, the Riemannian and affine structures need not be related; the connection is an independent degree of freedom locally given by non-metrizable Christoffel symbols [1]. In a general setting, the difference between the Christoffel symbols and those derived from the Levi-Civita connection is given by the non-metricity tensor and the torsion tensor [2].

Geometries with non-vanishing non-metricity have attracted renewed attention aimed at exploring the coupling to matter [3] as well as the geometric properties of new spacetime configurations (see e.g. [4] and references therein). On the other hand, gravity theories with non-vanishing torsion have been the subject of extensive study: there are models of the early universe in which torsion has a fundamental role as an alternative to inflation [5, 6]. The propagation of quantum fields on a spacetime with torsion has been analyzed in [7], and an example of the torsion field as a propagating degree of freedom can be found in [8]. Other models with non-metricity and torsion waves have been studied in [9] and [10]. A one-loop effective action in terms of the connection has been analyzed in [11]. Apart from the theoretical interest in non-Riemannian models of gravity, there are a variety of experiments which have been designed to measure the effects of torsion; for a quite comprehensive account we refer to [12]. There is also a substantial body of literature in the purely mathematical setting (we refer to [13-18], to cite just a few representative examples).

In this context we consider it useful to pursue the analysis of purely affine properties without regard to any possible Riemannian structure. The purpose of this work is to examine how the torsion impacts the geometry of a surface; we shall focus our attention on describing the effect of torsion on the associated Lie algebra of affine Killing vectors. As we do not use field equations, our results are model independent.

To ensure that the Lie algebra of affine Killing vectors is sufficiently rich, we shall assume that the surface in question is locally homogeneous; a complete classification of such local geometries is available [19, 20]. In addition, we believe that our study gives insight into the analysis of three- and higher-dimensional manifolds. This notwithstanding, theories of gravity with torsion in two dimensions constitute an active area on their own-for a review of its motivations and development see [21-23].

In the present paper we shall assume the underlying manifold in question is simply connected to facilitate the passage from local to global questions. The Lie algebra $\mathfrak{K}$ of affine Killing vector fields has played an important role in the study of surfaces which are torsion free; in this paper, we examine the relationship between the torsion and $\mathfrak{K}$. We say that a Lie sub-algebra $\mathfrak{K}_{0}$ of $\mathfrak{K}$ is effective if given any point $P$ of the underlying manifold, there exist $X_{i} \in \mathfrak{K}_{0}$ so that $\left\{X_{1}(P), X_{2}(P)\right\}$ are linearly independent. Since the underlying structure is assumed locally homogeneous and simply connected, $\mathfrak{K}$ is effective (see Hall [24] or Nomizu [25]). We refer to $[26,27]$ for recent examples where affine Killing vector fields have played an important role in the analysis.

We first present the fundamental definitions and properties of affine manifolds introducing torsion and the space of affine Killing vector fields. We focus on homogeneous affine surfaces and recall known results concerning their classification, in particular those related to affine Killing vector fields. We state the main result of the paper, namely the description of locally homogeneous affine surfaces in terms of the algebra of their affine Killing vectors.

\subsection{Affine surfaces, Christoffel symbols, and the torsion tensor}

An affine surface is a pair $\mathcal{M}=(M, \nabla)$ where $M$ is a smooth surface and $\nabla$ is a connection on the tangent bundle of $M$. In contrast to the notation adopted by some authors, we emphasize that we permit $\nabla$ to have torsion. Let $\partial_{k}:=\frac{\partial}{\partial x^{k}}$ in some system of local coordinates $\left(x^{1}, x^{2}\right)$ 
on $M$. We sum over repeated indices to express $\nabla_{i} \partial_{j}=\Gamma_{i j}^{k} \partial_{k}$; the connection is determined by the Christoffel symbols $\Gamma_{i j}^{k}$. For two vectors $X, Y$, let $T(X, Y):=\nabla_{X} Y-\nabla_{Y} X-[X, Y]$ be the torsion tensor; the components of the torsion tensor are expressed by

$$
T=\left(\mathrm{d} x^{i} \wedge \mathrm{d} x^{j}\right) \otimes\left(\Gamma_{i j}^{k}-\Gamma_{j i}^{k}\right) \partial_{k}=\left(\mathrm{d} x^{1} \wedge \mathrm{d} x^{2}\right) \otimes 4 T^{i} \partial_{i} \text { for } T^{i}:=\frac{1}{2}\left(\Gamma_{12}^{i}-\Gamma_{21}^{i}\right) .
$$

We say $\mathcal{M}$ is torsion free if $T=0$, i.e. if $\Gamma_{12}^{k}=\Gamma_{21}^{k}$ for $k=1,2$. There is a canonically associated torsion free connection ${ }^{0} \nabla:=\nabla-T$ with Christoffel symbols

$$
{ }^{0} \Gamma_{i j}^{k}:=\frac{1}{2}\left(\Gamma_{i j}^{k}+\Gamma_{j i}^{k}\right)
$$

The connection ${ }^{0} \nabla$ is in a certain sense the symmetric part of the connection $\nabla$ and the torsion $T$ is the anti-symmetric part. We let ${ }^{0} \mathcal{M}:=\left(M,{ }^{0} \nabla\right)$. Conversely, given an affine surface without torsion ${ }^{0} \mathcal{M}$ and a torsion tensor $T=\left(\mathrm{d} x^{1} \wedge \mathrm{d} x^{2}\right) \otimes\left(4 T^{i} \partial_{i}\right)$, we can perturb ${ }^{0} \mathcal{M}$ to define a surface ${ }^{T} \mathcal{M}$ with torsion $T$ by setting ${ }^{T} \nabla={ }^{0} \nabla+T$; the resulting Christoffel symbols are given by setting:

$$
\begin{array}{lll}
{ }^{T} \Gamma_{11}^{1}={ }^{0} \Gamma_{11}^{1}, & { }^{T} \Gamma_{11}^{2}={ }^{0} \Gamma_{11}^{2}, & { }^{T} \Gamma_{22}^{1}={ }^{0} \Gamma_{22}^{1}, \\
{ }^{T} \Gamma_{22}^{2}={ }^{0} \Gamma_{22}^{2}, & { }^{T} \Gamma_{12}^{1}={ }^{0} \Gamma_{12}^{1}+T^{1}, & { }^{T} \Gamma_{12}^{2}={ }^{0} \Gamma_{12}^{2}+T^{2}, \\
{ }^{T} \Gamma_{21}^{1}={ }^{0} \Gamma_{12}^{1}-T^{1}, & { }^{T} \Gamma_{21}^{2}={ }^{0} \Gamma_{12}^{2}-T^{2} . &
\end{array}
$$

These constructions are independent of the particular coordinate system chosen.

\subsection{Affine Killing vector fields}

Let $\mathcal{M}$ be an affine surface. A smooth vector field $X=v^{1} \partial_{1}+v^{2} \partial_{2}=\left(v^{1}, v^{2}\right)$ on an affine surface is said to be an affine Killing vector field if the Lie derivative of the connection with respect to the vector field $X$ vanishes or, equivalently (see Kobayashi and Nomizu [28, chapter $\mathrm{VI}]$ ), if the 8 affine Killing equations for $1 \leqslant i, j, k \leqslant 2$ are satisfied

$$
K_{i j}^{k}: \quad 0=\frac{\partial^{2} v^{k}}{\partial x^{i} \partial x^{j}}+v^{l} \frac{\partial \Gamma_{i j}^{k}}{\partial x^{l}}-\Gamma_{i j}^{l} \frac{\partial v^{k}}{\partial x^{l}}+\Gamma_{i l}^{k} \frac{\partial v^{l}}{\partial x^{j}}+\Gamma_{l j}^{k} \frac{\partial v^{l}}{\partial x^{i}} .
$$

The affine Killing equations form an over determined elliptic system of second-order partial differential equations. The Lie bracket makes the linear space $\mathfrak{K}(\mathcal{M})$ of affine Killing vector fields into a Lie algebra of dimension at most 6 since an affine Killing vector field is determined by $X(0)$ and $\nabla X(0)$.

\subsection{Homogeneous affine surfaces}

We say that a diffeomorphism from one affine surface to another is an affine map if it intertwines the two associated connections. We say that an affine manifold $\mathcal{M}$ is affine homogeneous if the Lie group of affine diffeomorphisms of $\mathcal{M}$ acts transitively; the corresponding local notion is defined similarly. To pass between local and global results, we shall assume henceforth that the underlying manifold $M$ is simply connected and locally affine homogeneous. In this setting, every affine Killing vector field which is locally defined extends to a globally defined affine Killing vector field. 
Opozda [20] classified the locally homogeneous affine surfaces without torsion; this classification was later extended to the case of surfaces with torsion by Arias-Marco and Kowalski [19]. We summarize their result as follows.

Theorem 1.1. If $\mathcal{M}$ is a locally homogeneous affine surface, possibly with torsion, then at least one of the following possibilities holds.

1. There exists a coordinate atlas for $M$ so that the Christoffel symbols of $\nabla$ are constant; $\mathcal{M}$ is said to be Type $\mathcal{A}$.

2. There exists a coordinate atlas for $M$ so that the Christoffel symbols have the form $\Gamma_{i j}^{k}=\left(x^{1}\right)^{-1} A_{i j}^{k}$, with $A_{i j}^{k}$ constant; $\mathcal{M}$ is said to be Type $\mathcal{B}$.

3. There exists a coordinate atlas for $M$ such that $\nabla$ is isomorphic to the Levi-Civita connection of the round sphere.

We say that $\mathcal{M}=\left(\mathbb{R}^{2}, \nabla\right)$ is a Type $\mathcal{A}$ model if the Christoffel symbols $\Gamma_{i j}^{k}$ are constant. If we identify $\mathbb{R}^{2}$ with the group of translations, then $\nabla$ is a Type $\mathcal{A}$ model if and only if $\nabla$ is left invariant. We can describe Type $\mathcal{A}$ models in terms of the algebra of translations in the plane. Let $\mathfrak{K}_{\mathcal{A}}:=\operatorname{span}\left\{\partial_{1}, \partial_{2}\right\}$. Then $\mathcal{M}=\left(\mathbb{R}^{2}, \nabla\right)$ is a Type $\mathcal{A}$ model if and only if $\mathfrak{K}_{\mathcal{A}} \subset \mathfrak{K}(\mathcal{M})$. We say that $\mathcal{N}=\left(\mathbb{R}^{+} \times \mathbb{R}\right)$ is a Type $\mathcal{B}$ model if $\Gamma_{i j}^{k}=\left(x^{1}\right)^{-1} A_{i j}^{k}$ for $A_{i j}^{k}$ constant. We identify $\mathbb{R}^{+} \times \mathbb{R}$ with the $a x+b$ group under the action $\left(x^{1}, x^{2}\right) \rightarrow\left(a x^{1}, a x^{2}+b\right) ;\left(\mathbb{R}^{+} \times \mathbb{R}, \nabla\right)$ is a Type $\mathcal{B}$ model if and only if $\nabla$ is left invariant under the natural action of the $a x+b$ group. By theorem 1.1, any locally homogeneous surface geometry is locally isomorphic to either a Type $\mathcal{A}$ model, a Type $\mathcal{B}$ model, or the round 2-sphere. We remark that there are geometries which admit both Type $\mathcal{A}$ and Type $\mathcal{B}$ structures. We also note that there are simply connected geometries with a Type $\mathcal{A}$ structure which are not affine equivalent to any open subset of a Type $\mathcal{A}$ model; more than one coordinate system is required for such geometries.

\subsection{The algebra of affine Killing vector fields for homogeneous surfaces}

Let $\mathcal{M}$ be a simply connected locally homogeneous affine surface. Fix a basepoint of $\mathcal{M}$. Define the following Lie algebra structures on $\mathbb{R}^{2}$ and $\mathbb{R}^{3}$ by the nonzero brackets:

$$
\begin{aligned}
\mathfrak{K}_{\mathcal{A}}:\left[e_{1}, e_{2}\right] & =0, \\
\mathfrak{K}_{\mathcal{B}}:\left[e_{1}, e_{2}\right] & =e_{1}, \\
\mathfrak{s o}(3):\left[e_{1}, e_{2}\right] & =e_{3},\left[e_{2}, e_{3}\right]=e_{1},\left[e_{3}, e_{1}\right]=e_{2}, \\
\mathfrak{s o}(2,1):\left[e_{1}, e_{2}\right] & =e_{1},\left[e_{2}, e_{3}\right]=e_{3},\left[e_{3}, e_{1}\right]=2 e_{2} .
\end{aligned}
$$

As already noted, $\mathfrak{K}_{\mathcal{A}}$ is the algebra of translations in the plane and $\mathfrak{K}_{\mathcal{B}}$ is the algebra of horizontal translations and dilatations in the upper half-plane. Following the notation of Patera et al [29], we define the following 4-dimensional Lie algebras by specifying their non-zero brackets:

$$
\begin{aligned}
A_{4,9}^{0}:\left[e_{2}, e_{3}\right] & =e_{1},\left[e_{1}, e_{4}\right]=e_{1},\left[e_{2}, e_{4}\right]=e_{2}, \\
A_{4,12}:\left[e_{1}, e_{3}\right] & =e_{1},\left[e_{2}, e_{3}\right]=e_{2},\left[e_{1}, e_{4}\right]=-e_{2},\left[e_{2}, e_{4}\right]=e_{1} .
\end{aligned}
$$

Let $\mathbb{A}_{6}$ be the 6-dimensional (6D) Lie algebra of the full affine group.

Recently Brozos-Vázquez et al [30] gave a quite different proof of theorem 1.1 by examining the affine Killing equations directly. Their result, from which theorem 1.1 follows, may be stated as follows. 
Lemma 1.2. Let $\mathcal{M}=(M, \nabla)$ be locally homogeneous and simply connected

1. There is an effective Lie subalgebra $\tilde{\mathfrak{K}}$ of $\mathfrak{K}(\mathcal{M})$ which is isomorphic to $\mathfrak{K}_{\mathcal{A}}, \mathfrak{K}_{\mathcal{B}}$, or $\mathfrak{s o}(3)$.

2. If $\tilde{\mathfrak{K}} \approx \mathfrak{K}_{\mathcal{A}}$, then there is a coordinate atlas so that $\Gamma_{i j}{ }^{k}$ are constant.

3. If $\tilde{\mathfrak{K}} \approx \mathfrak{K}_{\mathcal{B}}$, then there is a coordinate atlas so that $\Gamma_{i j}{ }^{k}=\left(x^{1}\right)^{-1} A_{i j}{ }^{k}$ for constant $A_{i j}{ }^{k}$.

4. If $\tilde{\mathfrak{K}} \approx \mathfrak{s o}(3)$, then there is a coordinate atlas where $\nabla$ is the Levi-Civita connection defined by the metric of the round sphere.

In this paper we will complete their analysis. Our main result is the following; it is implicit in the computations of Arias-Marco and Kowalski [19] but is not stated in this fashion there; our approach is quite different from theirs.

Theorem 1.3. Let $\mathcal{M}$ be a locally homogeneous simply connected affine surface with torsion.

1. Suppose $\mathcal{M}$ contains an effective Lie subalgebra which is isomorphic to $\mathfrak{K}_{\mathcal{A}}$. Then $\mathfrak{K}(\mathcal{M})$ is isomorphic to $\mathfrak{K}_{\mathcal{A}}$, to $\mathfrak{K}_{\mathcal{B}} \oplus \mathfrak{K}_{\mathcal{B}}$, to $A_{4,9}^{0}$, or to $A_{4,12}$.

2. Suppose $\mathcal{M}$ contains an effective Lie subalgebra which is isomorphic to $\mathfrak{K}_{\mathcal{B}}$. Then $\mathfrak{K}(\mathcal{M})$ is isomorphic to $\mathfrak{K}_{\mathcal{B}}$, to $\mathfrak{K}_{\mathcal{B}} \oplus \mathfrak{K}_{\mathcal{B}}$, to $A_{4,9}^{0}$, or to $\mathfrak{s o}(2,1)$.

3. Suppose $\mathcal{M}$ contains an effective Lie subalgebra which is isomorphic to $\mathfrak{s o}(3)$. Then $\mathcal{M}$ is without torsion and modeled on the round sphere.

\subsection{Outline of the paper}

The remainder of this paper is devoted to the proof of theorem 1.3. We begin in section 2 by establishing the following useful observation.

Lemma 1.4. Let $\mathcal{M}$ be an affine surface and let ${ }^{0} \mathcal{M}$ be the associated surface without torsion. Then $\mathfrak{K}(\mathcal{M}) \subseteq \mathfrak{K}\left({ }^{0} \mathcal{M}\right)$.

Brozos-Vázquez et al [31] and Gilkey and Valle-Regueiro [32] have classified, up to linear equivalence, all the Type $\mathcal{A}$ and Type $\mathcal{B}$ models without torsion where $\operatorname{dim}\{\mathfrak{K}\}>2$. Given an arbitrary model ${ }^{T} \mathcal{M}$ of Type $\mathcal{A}$ or Type $\mathcal{B}$ with torsion, we pass to the associated torsion free model ${ }^{0} \mathcal{M}$ and write down a basis for $\mathfrak{K}\left({ }^{0} \mathcal{M}\right)$. We then examine the affine Killing equations to determine which affine Killing vector fields on ${ }^{0} \mathcal{M}$ are affine Killing vector fields for $\mathcal{M}$. This then provides a classification of all the Type $\mathcal{A}$ and Type $\mathcal{B}$ models with torsion where $\operatorname{dim}\{\mathfrak{K}(\mathcal{M})\}>2$, which is of interest in its own right. Once this classification has been performed, we analyze the resulting Lie algebras to complete the proof of theorem 1.3. This analysis is performed in section 3 in the Type $\mathcal{A}$ setting and in section 4 in the Type $\mathcal{B}$ setting. The original analysis of Brozos-Vázquez et al [31] ignored the flat geometries as being uninteresting as they are in the torsion free setting. But once torsion is added, it is necessary to include these geometries as the flat geometries give rise to non-trivial geometries with torsion and for this the additional analysis of Gilkey and Valle-Regueiro [32] is required.

\section{Affine Killing equations in the presence of torsion}

In this section we give a proof of lemma 1.4. We also give two examples which help to understand the role of torsion in the affine Killing algebra. 
Let $\left(v^{1}, v^{2}\right) \in \mathfrak{K}\left({ }^{T} \mathcal{M}\right)$. Call ${ }^{T} K_{i j}^{k}$ the r.h.s of equation (2) when the Christoffel symbols involve a torsion $T$. The corresponding equation for the symmetrized part of the Christoffel symbols is ${ }^{0} K_{i j}^{k}$. A direct computation gives

$$
{ }^{T} K_{i j}^{k}={ }^{0} K_{i j}^{k}+v^{l} \frac{\partial T_{i j}^{k}}{\partial x^{l}}-T_{i j}^{l} \frac{\partial v^{k}}{\partial x^{l}}+T_{i l}^{k} \frac{\partial v^{l}}{\partial x^{j}}+T_{l j}^{k} \frac{\partial v^{l}}{\partial x^{i}}=0 .
$$

Taking $i=j$ in the last expression gives ${ }^{T} K_{i i}^{k}={ }^{0} K_{i i}^{k}=0$. To obtain a similar result for the nondiagonal elements consider the equations

$$
\begin{aligned}
& { }^{T} K_{i j}^{k}={ }^{0} K_{i j}^{k}+v^{l} \frac{\partial T_{i j}^{k}}{\partial x^{l}}-T_{i j}^{l} \frac{\partial v^{k}}{\partial x^{l}}+T_{i l}^{k} \frac{\partial v^{l}}{\partial x^{j}}+T_{l j}^{k} \frac{\partial v^{l}}{\partial x^{i}}=0, \\
& { }^{T} K_{j i}^{k}={ }^{0} K_{j i}^{k}+v^{l} \frac{\partial T_{j i}^{k}}{\partial x^{l}}-T_{j i}^{l} \frac{\partial v^{k}}{\partial x^{l}}+T_{j l}^{k} \frac{\partial v^{l}}{\partial x^{i}}+T_{l i}^{k} \frac{\partial v^{l}}{\partial x^{j}}=0 .
\end{aligned}
$$

Adding these we have $0={ }^{T} K_{i j}^{k}+{ }^{T} K_{j i}^{k}={ }^{0} K_{i j}^{k}+{ }^{0} K_{j i}^{k}$. Since the Christoffel symbols for $T=0$ are symmetric, ${ }^{0} K_{i j}^{k}={ }^{0} K_{j i}^{k}=0$. Lemma 1.4 follows.

Example 2.1. Let $\mathcal{M}_{1}^{4}$ be the Type $\mathcal{A}$ surface without torsion defined by the Christoffel symbols $\Gamma_{11}^{1}=-1, \Gamma_{12}^{1}=1, \Gamma_{22}^{1}=0, \Gamma_{11}^{2}=0, \Gamma_{12}^{2}=0$ and $\Gamma_{22}^{2}=2$. Let $0 \neq T=\left(T^{1}, T^{2}\right) \in \mathbb{R}^{2}$. We shall see presently that $\operatorname{dim}\{\mathfrak{K}(\mathcal{M})\}=4$, that $\operatorname{dim}\left\{\mathfrak{K}\left({ }^{T} \mathcal{M}\right)\right\}=4$ if $T^{2}=0$. This shows that the equality in lemma 1.4 can hold.

Example 2.2. Given a torsion free manifold which is locally homogeneous, the perturbed manifold need not be homogeneous. Consider the type $\mathcal{A}$ surface $\mathcal{M}_{1}^{6}$ defined by the Christoffel symbols $\Gamma_{11}^{1}=1, \Gamma_{12}^{1}=0, \Gamma_{22}^{1}=0, \Gamma_{11}^{2}=0, \Gamma_{12}^{2}=1$ and $\Gamma_{22}^{2}=0$, with $\operatorname{dim}\left\{\mathfrak{K}\left(\mathcal{M}_{1}^{6}\right)\right\}=6$. Perturb it by adding a type $\mathcal{B}$ torsion $T$ where $T^{1}=0$ and $T^{2}=t^{2} / x^{1} \neq 0$. The resulting structure has

$$
\mathfrak{K}\left({ }^{T} \mathcal{M}_{1}^{6}\right)=\operatorname{span}\left\{\partial_{2}, x^{2} \partial_{2}, \mathrm{e}^{-x^{1}} \partial_{2}\right\}
$$

This algebra has no effective subalgebras for all $\left(x^{1}, x^{2}\right)$ and hence the surface is not homogeneous. Now perturb it by adding a type $\mathcal{B}$ torsion $T$ with $T^{1}=t^{1} / x^{1} \neq 0$ and $T^{2}=0$. The resulting structure has $\mathfrak{K}\left({ }^{T} \mathcal{M}_{1}^{6}\right)=\operatorname{span}\left\{\partial_{2}\right\}$. This example shows that the addition of torsion to a homogeneous, torsion free surface does not necessarily give a homogeneous surface.

\section{Type $\mathcal{A}$ surfaces with torsion}

In this section we obtain the spaces of affine Killing vector fields for Type $\mathcal{A}$ models. This gives the algebras of theorem 1.3(1).

Parametrize the set of Type $\mathcal{A}$ models by setting $\mathcal{M}(\vec{\xi}):=\left(\mathbb{R}^{2}, \nabla^{\mathcal{A}}(\vec{\xi})\right)$ for $\xi \in \mathbb{R}^{8}$ where the Christoffel symbols of $\nabla^{\mathcal{A}}(\vec{\xi})$ are given by:

$$
\begin{aligned}
& \Gamma_{11}^{1}=\xi_{1}, \Gamma_{11}^{2}=\xi_{2}, \Gamma_{12}{ }^{1}=\xi_{3}, \Gamma_{12}^{2}=\xi_{4}, \\
& \Gamma_{21}{ }^{1}=\xi_{5}, \Gamma_{21}{ }^{2}=\xi_{6}, \Gamma_{22}{ }^{1}=\xi_{7}, \Gamma_{22}{ }^{2}=\xi_{8} .
\end{aligned}
$$

The torsion free models $\mathcal{M}(\vec{\xi})$ form a $6 \mathrm{D}$ subspace where $\xi_{3}=\xi_{5}$ and $\xi_{4}=\xi_{6}$. The general linear group $\operatorname{GL}(2, \mathbb{R})$ acts on the space of Type $\mathcal{A}$ models by change of basis and defines thereby a linear representation of $\operatorname{GL}(2, \mathbb{R})$ on $\mathbb{R}^{8}$. We say that two Type $\mathcal{A}$ models are linearly equivalent if they lie in the same orbit of this representation. The works $[31,32]$ mentioned 
previously classify all Type $\mathcal{A}$ torsion free models up to linear equivalence. We restrict this classification to those torsion free models where $\operatorname{dim}\{\mathfrak{K}\}>2$ to obtain models $\mathcal{M}_{i}^{j}(\star ; 0)$ where there is an auxiliary parameter $\star$ in certain examples. If $j=6$, then $\operatorname{dim}\left\{\mathfrak{K}\left(\mathcal{M}_{i}^{j}(\star ; 0)\right)\right\}=6$ and if $j=4$, then $\operatorname{dim}\left\{\mathfrak{K}\left(\mathcal{M}_{i}^{j}(\star ; 0)\right)\right\}=4$. We then add torsion to obtain models $\mathcal{M}_{i}^{j}(\star ; T)$; we no longer have, of course, that $\operatorname{dim}\left\{\mathfrak{K}\left(\mathcal{M}_{i}^{j}(\star ; T)\right)\right\}=j$ if $T \neq 0$. Still, it seemed useful to keep the notation since ${ }^{0} \mathcal{M}_{i}^{j}(\star ; T)=\mathcal{M}_{i}^{j}(\star ; 0)$. We have that $\mathcal{M}_{i}^{j}(\star ; T)$ and $\mathcal{M}_{k}^{\ell}(\star ; \tilde{T})$ are not linearly equivalent for $(i, j) \neq(k, \ell)$. Within a given class defined by $(i, j)$ determining the precise set of representatives under linear equivalence is considerably more delicate and we have not attempted such a finer classification.

We now establish the main result of the paper. To obtain assertion (1) in theorem 1.3 we will compute the Lie algebras of affine Killing vector fields for all the models $\mathcal{M}_{i}^{j}(\star ; T)$. We first write down a basis for $\mathfrak{K}\left(\mathcal{M}_{i}^{j}(\star ; 0)\right)$ and then examine the effect of the torsion tensor on the affine Killing equations to derive the following result.

Lemma 3.1. Let $\mathcal{M}$ be a Type $\mathcal{A}$ model with torsion tensor $T=\left(T^{1}, T^{2}\right)$ so that $\operatorname{dim}\{\mathfrak{K}(\mathcal{M})\}>2$. Then $\mathcal{M}$ is linearly equivalent to one of the following surfaces with the values of T listed; $\mathfrak{K}(\mathcal{M})=\operatorname{span}\left\{\partial_{1}, \partial_{2}\right\}$ for other values of $T$.

1. Let $\mathcal{M}_{0}^{6}(T):=\mathcal{M}\left(0,0, T^{1}, T^{2},-T^{1},-T^{2}, 0,0\right)$. Then

(a) $\mathfrak{K}\left(\mathcal{M}_{0}^{6}(0)\right)=\operatorname{span}\left\{\partial_{1}, \partial_{2}, x^{1} \partial_{1}, x^{1} \partial_{2}, x^{2} \partial_{1}, x^{2} \partial_{2}\right\}$.

(b) $\mathfrak{K}\left(\mathcal{M}_{0}^{6}(T)\right)=\operatorname{span}\left\{\partial_{1}, \partial_{2}, x^{1}\left(T^{1} \partial_{1}+T^{2} \partial_{2}\right), x^{2}\left(T^{1} \partial_{1}+T^{2} \partial_{2}\right)\right\}$ if $T \neq 0$.

2. Let $\mathcal{M}_{1}^{6}(T):=\mathcal{M}\left(1,0, T^{1}, 1+T^{2},-T^{1}, 1-T^{2}, 0,0\right)$. Then $T^{l}=0$ and

(a) $\mathfrak{K}\left(\mathcal{M}_{1}^{6}(0,0)\right)=\operatorname{span}\left\{\partial_{1}, \mathrm{e}^{-x^{1}}\left(\partial_{1}-x^{2} \partial_{2}\right), x^{2} \partial_{2}, x^{2}\left(\partial_{1}-x^{2} \partial_{2}\right), \partial_{2}, \mathrm{e}^{-x^{1}} \partial_{2}\right\}$.

(b) $\mathfrak{K}\left(\mathcal{M}_{1}^{6}\left(0, T^{2}\right)\right)=\operatorname{span}\left\{\partial_{1}, \partial_{2}, x^{2} \partial_{2}, \mathrm{e}^{-x^{1}} \partial_{2}\right\}$ if $T^{2} \neq 0$.

3. Let $\mathcal{M}_{2}^{6}(T):=\mathcal{M}\left(-1,0, T^{1}, T^{2},-T^{1},-T^{2}, 0,1\right)$. Then $T^{1} T^{2}=0$ and

(a) $\mathfrak{K}\left(\mathcal{M}_{2}^{6}(0)\right)=\operatorname{span}\left\{\partial_{1}, \mathrm{e}^{x^{1}} \partial_{1}, \mathrm{e}^{x^{1}+x^{2}} \partial_{1}, \partial_{2}, \mathrm{e}^{-x^{1}-x^{2}} \partial_{2}, \mathrm{e}^{-x^{2}} \partial_{2}\right\}$.

(b) $\mathfrak{K}\left(\mathcal{M}_{2}^{6}(T)\right)=\operatorname{span}\left\{\partial_{1}, \partial_{2}, \mathrm{e}^{-x^{1}-x^{2}} \partial_{2}, \mathrm{e}^{-x^{2}} \partial_{2}\right\}$ if $T^{1}=0, T^{2} \neq 0$.

(c) $\mathfrak{K}\left(\mathcal{M}_{2}^{6}(T)\right)=\operatorname{span}\left\{\partial_{1}, \mathrm{e}^{x^{1}} \partial_{1}, \mathrm{e}^{x^{1}+x^{2}} \partial_{1}, \partial_{2}\right\}$ if $T^{1} \neq 0, T^{2}=0$.

4. Let $\mathcal{M}_{3}^{6}(T):=\mathcal{M}\left(0,0, T^{1}, T^{2},-T^{1},-T^{2}, 0,1\right)$. Then $T^{1} T^{2}=0$ and

(a) $\mathfrak{K}\left(\mathcal{M}_{3}^{6}(0)\right)=\operatorname{span}\left\{\partial_{1}, x^{1} \partial_{1}, \mathrm{e}^{x^{2}} \partial_{1}, \partial_{2}, \mathrm{e}^{-x^{2}} \partial_{2}, x^{1} \mathrm{e}^{-x^{2}} \partial_{2}\right\}$.

(b) $\mathfrak{K}\left(\mathcal{M}_{3}^{6}(T)\right)=\operatorname{span}\left\{\partial_{1}, \partial_{2}, \mathrm{e}^{-x^{2}} \partial_{2}, x^{1} \mathrm{e}^{-x^{2}} \partial_{2}\right\}$ if $T^{1}=0, T^{2} \neq 0$.

(c) $\mathfrak{K}\left(\mathcal{M}_{3}^{6}(T)\right)=\operatorname{span}\left\{\partial_{1}, \partial_{2}, x^{1} \partial_{1}, \mathrm{e}^{x^{2}} \partial_{1}\right\}$ if $T^{1} \neq 0, T^{2}=0$.

5. Let $\mathcal{M}_{4}^{6}(T):=\mathcal{M}\left(0,0, T^{1}, T^{2},-T^{1},-T^{2}, 1,0\right)$. Then $T^{2}=0$ and

(a) $\mathfrak{K}\left(\mathcal{M}_{4}^{6}(0)\right)=\operatorname{span}\left\{\partial_{1}, \partial_{2},\left(x^{1}+\frac{1}{2}\left(x^{2}\right)^{2}\right) \partial_{1}, x^{2} \partial_{1}\right.$, $\left.\left(-x^{1} x^{2}-\frac{1}{2}\left(x^{2}\right)^{3}\right) \partial_{1}+\left(x^{1}+\frac{1}{2}\left(x^{2}\right)^{2}\right) \partial_{2},-\left(x^{2}\right)^{2} \partial_{1}+x^{2} \partial_{2}\right\}$.

(b) $\mathfrak{K}\left(\mathcal{M}_{4}^{6}\left(T^{1}, 0\right)\right)=\operatorname{span}\left\{\partial_{1}, \partial_{2},\left(x^{1}+\frac{1}{2}\left(x^{2}\right)^{2}\right) \partial_{1}, x^{2} \partial_{1}\right\}$ for $T^{1} \neq 0$.

6. Let $\mathcal{M}_{5}^{6}(T)=\mathcal{M}\left(1,0, T^{1}, 1+T^{2},-T^{1}, 1-T^{2},-1,0\right)$. Then $T=0$ and $\mathfrak{K}\left(\mathcal{M}_{5}^{6}(0)\right)=\operatorname{span}\left\{\partial_{1}, \partial_{2}, \cos \left(2 x^{2}\right) \partial_{1}-\sin \left(2 x^{2}\right) \partial_{2}, \sin \left(2 x^{2}\right) \partial_{1}+\cos \left(2 x^{2}\right) \partial_{2}\right.$ $\left.\mathrm{e}^{-x^{1}}\left(\cos \left(x^{2}\right) \partial_{1}-\sin \left(x^{2}\right) \partial_{2}\right), \mathrm{e}^{-x^{1}}\left(\sin \left(x^{2}\right) \partial_{1}+\cos \left(x^{2}\right) \partial_{2}\right)\right\}$.

7. Let $\mathcal{M}_{1}^{4}(T):=\mathcal{M}\left(-1,0,1+T^{1}, T^{2}, 1-T^{1},-T^{2}, 0,2\right)$. Then $T^{2}=0$ and $\mathfrak{K}\left(\mathcal{M}_{1}^{4}\left(T^{1}, 0\right)\right)=\operatorname{span}\left\{\partial_{1}, \partial_{2}, \mathrm{e}^{x^{1}} \partial_{1}, x^{2} \mathrm{e}^{x^{1}} \partial_{1}\right\}$

8. Let $\mathcal{M}_{2}^{4}(c ; T):=\mathcal{M}\left(-1,0, c+T^{1}, T^{2}, c-T^{1},-T^{2}, 0,1+2 c\right)$ for $c \neq 0,-1$. Then $T^{2}=0$

and $\mathfrak{K}\left(\mathcal{M}_{2}^{4}\left(c ;\left(T^{1}, 0\right)\right)\right)=\operatorname{span}\left\{\partial_{1}, \partial_{2}, \mathrm{e}^{x^{1}} \partial_{1}, \mathrm{e}^{x^{1}+x^{2}} \partial_{1}\right\}$.

9. Let $\mathcal{M}_{3}^{4}(c ; T):=\mathcal{M}\left(0,0, c+T^{1}, T^{2}, c-T^{1},-T^{2}, 0,1+2 c\right)$ for $c \neq 0,-1$. Then $T^{2}=0$ and $\mathfrak{K}\left(\mathcal{M}_{3}^{4}\left(c ;\left(T^{1}, 0\right)\right)\right)=\operatorname{span}\left\{\partial_{1}, \partial_{2}, \mathrm{e}^{x^{2}} \partial_{1}, x^{1} \partial_{1}\right\}$.

10. Let $\mathcal{M}_{4}^{4}(c ; T):=\mathcal{M}\left(0,0,1+T^{1}, T^{2}, 1-T^{1},-T^{2}, c, 2\right)$. Then $T^{2}=0$ and $\mathfrak{K}\left(\mathcal{M}_{4}^{4}\left(c ;\left(T^{1}, 0\right)\right)\right)=\operatorname{span}\left\{\partial_{1}, \partial_{2},\left(x^{1}+\frac{c}{2}\left(x^{2}\right)^{2}\right) \partial_{1}, x^{2} \partial_{1}\right\}$. 


$$
\begin{aligned}
& \text { 11. Let } \mathcal{M}_{5}^{4}(c ; T):=\mathcal{M}\left(1,0, T^{1}, T^{2},-T^{1},-T^{2}, 1+c^{2}, 2 c\right) \text {. Then } T^{2}=0 \text { and } \\
& \mathfrak{K}\left(\mathcal{M}_{5}^{4}\left(c ;\left(T^{1}, 0\right)\right)\right)=\operatorname{span}\left\{\partial_{1}, \partial_{2}, \mathrm{e}^{-x^{1}+c x^{2}} \cos x^{2} \partial_{1}, \mathrm{e}^{-x^{1}+c x^{2}} \sin x^{2} \partial_{1}\right\} \text {. }
\end{aligned}
$$

One now performs a careful examination of the Lie algebras of lemma 3.1 to determine their isomorphism type. This leads to the following classification result from which theorem 1.3(1) follows:

Lemma 3.2. $\quad$ Adopt the notation established in lemma 3.1. Let $\mathcal{M}$ be a Type $\mathcal{A}$ model with torsion. Generically, $\mathfrak{K}(\mathcal{M})=\mathfrak{K}_{\mathcal{A}}$. Let $\varepsilon \neq 0$ and let $\left(\varepsilon_{1}, \varepsilon_{2}\right) \neq(0,0)$. If $\operatorname{dim}\{\mathfrak{K}(\mathcal{M})\}>2$, then $\mathfrak{K}(\mathcal{M})$ has one of the following structures.

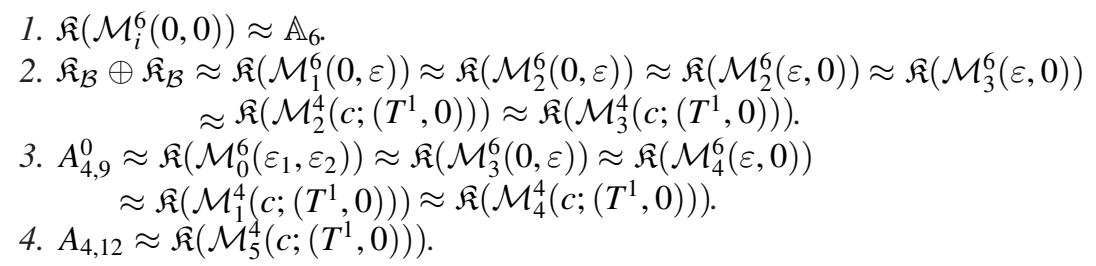

\section{Type $\mathcal{B}$ surfaces with torsion}

We proceed in a similar fashion in the Type $\mathcal{B}$ setting. We parametrize the set of Type $\mathcal{B}$ models by setting $\mathcal{N}(\vec{\xi}):=\left(\mathbb{R}^{+} \times \mathbb{R}, \nabla^{\mathcal{B}}(\vec{\xi})\right)$ where the Christoffel symbols take the form:

$$
\begin{aligned}
& \Gamma_{11}{ }^{1}=\left(x^{1}\right)^{-1} \xi_{1}, \Gamma_{11}{ }^{2}=\left(x^{1}\right)^{-1} \xi_{2}, \Gamma_{12}{ }^{1}=\left(x^{1}\right)^{-1} \xi_{3}, \Gamma_{12}{ }^{2}=\left(x^{1}\right)^{-1} \xi_{4}, \\
& \Gamma_{21}{ }^{1}=\left(x^{1}\right)^{-1} \xi_{5}, \Gamma_{21}{ }^{2}=\left(x^{1}\right)^{-1} \xi_{6}, \Gamma_{22}{ }^{1}=\left(x^{1}\right)^{-1} \xi_{7}, \Gamma_{22}{ }^{2}=\left(x^{1}\right)^{-1} \xi_{8} .
\end{aligned}
$$

The structure group for the set of Type $\mathcal{B}$ models is not the full general linear group but rather the $a x+b$ group acting by the shear $\left(x^{1}, x^{2}\right) \rightarrow\left(x^{1}, b x^{1}+a x^{2}\right)$; again we say two Type $\mathcal{B}$ models are linearly equivalent if they are in the same orbit of the induced linear action on $\mathbb{R}^{8}$. The work of $[31,32]$ mentioned previously does not provide a full classification of all the Type $\mathcal{B}$ models without torsion up to linear equivalence. It does suffice, for our purposes, in that it does classify the torsion free Type $\mathcal{B}$ models with $\operatorname{dim}\{\mathfrak{K}\}>2$ by providing models $\mathcal{N}_{i}^{j}(\star ; 0)$ where $\star$ is an auxiliary parameter present in some instances. Of particular interest are the geometries $\mathcal{N}_{3}^{3}$, which is the Lorentzian hyperbolic plane, and $\mathcal{N}_{4}^{3}$, which is the hyperbolic plane. The geometries $\mathcal{N}_{i}^{4}(\star ; 0)$ are also Type $\mathcal{A}$ geometries. The torsion tensors are, of course, quite different. The geometries $\mathcal{N}_{i}^{6}(\star ; 0)$ are flat. The proof of lemma 4.1 now follows by first writing down a basis for $\mathfrak{K}\left(\mathcal{N}_{i}^{j}(\star ; 0)\right)$ and then examining the effect of the torsion tensor on the affine Killing equations.

Lemma 4.1. Let $X:=x^{1} \partial_{1}+x^{2} \partial_{2}$. Let $\mathcal{N}$ be a Type $\mathcal{B}$ model with torsion tensor $T=\left(T^{1}, T^{2}\right)$ so that $\operatorname{dim}\{\mathfrak{K}(\mathcal{N})\}>2$. Then $\mathcal{N}$ is linearly equivalent to one of the following surfaces with the values of T listed; $\mathfrak{K}(\mathcal{N})=\operatorname{span}\left\{X, \partial_{2}\right\}$ for other values of $T$.

1. Let $\mathcal{N}_{0}^{6}(T):=\mathcal{N}\left(0,0, T^{1}, T^{2},-T^{1},-T^{2}, 0,0\right)$. Then

(a) $\mathfrak{K}\left(\mathcal{N}_{0}^{6}(0)\right)=\operatorname{span}\left\{\partial_{1}, \partial_{2}, x^{1} \partial_{1}, x^{1} \partial_{2}, x^{2} \partial_{1}, x^{2} \partial_{2}\right\}$.

(b) $\mathfrak{K}\left(\mathcal{N}_{0}^{6}\left(0, T^{2}\right)\right)=\operatorname{span}\left\{X, \partial_{2}, x^{1} \partial_{2}, x^{2} \partial_{2}\right\}$ if $T^{2} \neq 0$.

2. Let $\mathcal{N}_{1}^{6}( \pm ; T):=\mathcal{N}\left(1,0, T^{1}, T^{2},-T^{1},-T^{2}, \pm 1,0\right)$. Then $T=0$ and

$\mathcal{K}\left(\mathcal{N}_{1}^{6}( \pm, 0)\right)=\operatorname{span}\left\{X, \partial_{2}, \frac{1}{x^{1}} \partial_{1}, \frac{x^{2}}{x^{1}} \partial_{1}, \frac{\left(x^{1}\right)^{2} \pm\left(x^{2}\right)^{2}}{x^{1}} \partial_{1}, \frac{-x^{2}\left(\left(x^{2}\right)^{2} \pm\left(x^{1}\right)^{2}\right)}{x^{1}} \partial_{1}+\left(\left(x^{1}\right)^{2} \pm\left(x^{2}\right)^{2}\right) \partial_{2}\right\}$.

3. Let $\mathcal{N}_{2}^{6}(c ; T):=\mathcal{N}\left(-1+c, 0, T^{1}, c+T^{2},-T^{1}, c-T^{2}, 0,0\right)$ for $c \neq 0$. Then $T^{1} T^{2}=0$ 
and

(a) $\mathfrak{K}\left(\mathcal{N}_{2}^{6}(c ; 0)\right)=\operatorname{span}\left\{x^{1} \partial_{1}, x^{2} \partial_{2}, \partial_{2},\left(x^{1}\right)^{-c} \partial_{2},\left(x^{1}\right)^{-c}\left(x^{1} \partial_{1}-c x^{2} \partial_{2}\right), x^{2}\left(x^{1} \partial_{1}-c x^{2} \partial_{2}\right)\right\}$.

(b) $\mathfrak{K}\left(\mathcal{N}_{2}^{6}\left(c ;\left(0, T^{2}\right)\right)\right)=\operatorname{span}\left\{X, \partial_{2}, x^{2} \partial_{2},\left(x^{1}\right)^{-c} \partial_{2}\right\}$ if $T^{2} \neq 0$.

(c) $\mathfrak{K}\left(\mathcal{N}_{2}^{6}\left(-\frac{1}{2} ;\left(T^{1}, 0\right)\right)\right)=\operatorname{span}\left\{X, \partial_{2}, x^{2}\left(x^{1} \partial_{1}+\frac{1}{2} x^{2} \partial_{2}\right)\right\}$ if $T^{1} \neq 0$.

4. Let $\mathcal{N}_{3}^{6}(T):=\mathcal{N}\left(-2,1, T^{1},-1+T^{2},-T^{1},-1-T^{2}, 0,0\right)$. Then $T^{1}=0$ and

(a) $\mathfrak{K}\left(\mathcal{N}_{3}^{6}(0)\right)=\operatorname{span}\left\{X, \partial_{2}, x^{1} \partial_{2},\left(x^{2}+x^{1} \log x^{1}\right) \partial_{2}\right.$,

$\left.-\left(x^{1}\right)^{2} \partial_{1}+x^{1}\left(x^{1}-x^{2}\right) \partial_{2},\left(x^{2}+x^{1} \log x^{1}\right)\left(-x^{1} \partial_{1}+\left(x^{1}-x^{2}\right) \partial_{2}\right)\right\}$.

(b) $\mathfrak{K}\left(\mathcal{N}_{3}^{6}\left(0, T^{2}\right)\right)=\operatorname{span}\left\{X, \partial_{2}, x^{1} \partial_{2},\left(x^{2}+x^{1} \log x^{1}\right) \partial_{2}\right\}$ if $T^{2} \neq 0$.

5. Let $\mathcal{N}_{4}^{6}(T):=\mathcal{N}\left(0,1, T^{1}, T^{2},-T^{1},-T^{2}, 0,0\right)$. Then $T^{l}=0$ and

(a) $\mathfrak{K}\left(\mathcal{N}_{4}^{6}(0)\right)=\operatorname{span}\left\{X, \partial_{2}, x^{1} \partial_{2},\left(x^{2}+x^{1} \log x^{1}\right) \partial_{2}\right.$,

$$
\left.\partial_{1}-\left(1+\log x^{1}\right) \partial_{2},\left(x^{2}+x^{1} \log x^{1}\right)\left(\partial_{1}-\left(1+\log x^{1}\right) \partial_{2}\right)\right\} .
$$

(b) $\mathfrak{K}\left(\mathcal{N}_{4}^{6}\left(0, T^{2}\right)\right)=\operatorname{span}\left\{X, \partial_{2}, x^{1} \partial_{2},\left(x^{2}+x^{1} \log x^{1}\right) \partial_{2}\right\}$ if $T^{2} \neq 0$.

6. Let $\mathcal{N}_{5}^{6}(T):=\mathcal{N}\left(-1,0, T^{1}, T^{2},-T^{1},-T^{2}, 0,0\right)$. Then $T^{1}=0$ and

(a) $\mathfrak{K}\left(\mathcal{N}_{5}^{6}(0)\right)=\operatorname{span}\left\{X, \partial_{2}, x^{2} \partial_{2}, x^{1} x^{2} \partial_{1}, \log x^{1} \partial_{2}, x^{1} \log x^{1} \partial_{1}\right\}$.

(b) $\mathfrak{K}\left(\mathcal{N}_{5}^{6}\left(0, T^{2}\right)\right)=\operatorname{span}\left\{X, \partial_{2}, x^{2} \partial_{2}, \log x^{1} \partial_{2}\right\}$ if $T^{2} \neq 0$.

7. Let $\mathcal{N}_{6}^{6}(c ; T):=\mathcal{N}\left(c, 0, T^{1}, T^{2},-T^{1},-T^{2}, 0,0\right)$ for $c \neq 0,-1$. Then $T^{l}=0$ and

(a) $\mathfrak{K}\left(\mathcal{N}_{6}^{6}(c ; 0)\right)=\operatorname{span}\left\{X, \partial_{2}, x^{2} \partial_{2},\left(x^{1}\right)^{-c} \partial_{1},\left(x^{1}\right)^{-c} x^{2} \partial_{1},\left(x^{1}\right)^{c+1} \partial_{2}\right\}$.

(b) $\mathfrak{K}\left(\mathcal{N}_{6}^{6}\left(c ;\left(0, T^{2}\right)\right)\right)=\operatorname{span}\left\{X, \partial_{2}, x^{2} \partial_{2},\left(x^{1}\right)^{c+1} \partial_{2}\right\}$ if $T^{2} \neq 0$.

8. Let $\mathcal{N}_{1}^{4}(\kappa ; T):=\mathcal{N}\left(2 \kappa, 1, T^{1}, T^{2}+\kappa,-T^{1},-T^{2}+\kappa, 0,0\right)$ for $\kappa \neq 0,-1$. Then $T^{l}=0$ and $\mathfrak{K}\left(\mathcal{N}_{1}^{4}\left(\kappa ;\left(0, T^{2}\right)\right)\right)=\operatorname{span}\left\{X, \partial_{2}, x^{1} \partial_{2}, x^{1}\left(\partial_{1}-\log x^{1} \partial_{2}\right)\right\}$.

9. Let $\mathcal{N}_{2}^{4}(\kappa, \theta ; T):=\mathcal{N}\left(-1+\theta+2 \kappa, 0, T^{1}, T^{2}+\kappa,-T^{1},-T^{2}+\kappa, 0,0\right)$ for $\theta \neq 0$ and $\kappa(\kappa+\theta) \neq 0$. Then $T^{l}=0$ and $\mathfrak{K}\left(\mathcal{N}_{2}^{4}\left(\kappa, \theta ;\left(0, T^{2}\right)\right)\right)=\operatorname{span}\left\{X, \partial_{2}, x^{2} \partial_{2},\left(x^{1}\right)^{\theta} \partial_{2}\right\}$.

10. Let $\mathcal{N}_{3}^{4}(c ; T):=\mathcal{N}\left(2 c-1,0, T^{1}, T^{2}+c,-T^{1},-T^{2}+c, 0,0\right)$ for $c \neq 0$. Then $T^{l}=0$ and $\mathfrak{K}\left(\mathcal{N}_{3}^{4}\left(c ;\left(0, T^{2}\right)\right)\right)=\operatorname{span}\left\{X, \partial_{2}, x^{2} \partial_{2}, \log x^{1} \partial_{2}\right\}$.

11. Let $\mathcal{N}_{1}^{3}( \pm ; T):=\mathcal{N}\left(-\frac{3}{2}, 0, T^{1},-\frac{1}{2}+T^{2},-T^{1},-\frac{1}{2}-T^{2}, \pm \frac{1}{2}, 0\right)$. Then $T^{2}=0$ and $\mathfrak{K}\left(\mathcal{N}_{1}^{3}\left( \pm ;\left(T^{1}, 0\right)\right)\right)=\operatorname{span}\left\{X, \partial_{2}, x^{2}\left(2 x^{1} \partial_{1}+x^{2} \partial_{2}\right)\right\}$.

12. Let $\mathcal{N}_{2}^{3}(c ; T):=\mathcal{N}\left(-\frac{3}{2}, 0,1+T^{1},-\frac{1}{2}+T^{2}, 1-T^{1},-\frac{1}{2}-T^{2}, c, 2\right)$. Then $T^{2}=0$ and $\mathfrak{K}\left(\mathcal{N}_{2}^{3}\left(c ;\left(T^{1}, 0\right)\right)\right)=\operatorname{span}\left\{X, \partial_{2}, x^{2}\left(2 x^{1} \partial_{1}+x^{2} \partial_{2}\right)\right\}$.

13. Let $\mathcal{N}_{3}^{3}(T):=\mathcal{N}\left(-1,0, T^{1},-1+T^{2},-T^{1},-1-T^{2},-1,0\right)$. Then $T=0$ and $\mathfrak{K}\left(\mathcal{N}_{3}^{3}(T)\right)=\operatorname{span}\left\{X, \partial_{2}, 2 x^{1} x^{2} \partial_{1}+\left(\left(x^{2}\right)^{2}+\left(x^{1}\right)^{2}\right) \partial_{2}\right\}$.

14. Let $\mathcal{N}_{4}^{3}(T):=\mathcal{N}\left(-1,0, T^{1},-1+T^{2},-T^{1},-1-T^{2}, 1,0\right)$. Then $T=0$ and $\mathfrak{K}\left(\mathcal{N}_{4}^{3}(0)\right)=\operatorname{span}\left\{X, \partial_{2}, 2 x^{1} x^{2} \partial_{1}+\left(\left(x^{2}\right)^{2}-\left(x^{1}\right)^{2}\right) \partial_{2}\right\}$.

One now performs a careful examination of the Lie algebras of lemma 4.1 to determine their isomorphism type. This leads to the following classification result from which theorem 1.3(2) follows:

Lemma 4.2. Adopt the notation established in lemma 4.1. Let $\mathcal{N}$ be a Type $\mathcal{B}$ model with torsion. Generically, $\mathfrak{K}(\mathcal{N})$ is 2-dimensional $(2 D)$ and is isomorphic to the $2 D$ non-Abelian Lie algebra $\mathfrak{K}_{\mathcal{B}}$. Let $\varepsilon \neq 0$. If $\operatorname{dim}\{\mathfrak{K}(\mathcal{N})\}>2$, then $\mathfrak{K}(\mathcal{N})$ has one of the following structures.

1. $\mathfrak{K}\left(\mathcal{N}_{i}^{6}(0,0)\right) \approx \mathbb{A}_{6}$.

2. $\mathfrak{K}_{\mathcal{B}} \oplus \mathfrak{K}_{\mathcal{B}} \approx \mathfrak{K}\left(\mathcal{N}_{0}^{6}((0, \varepsilon))\right) \approx \mathfrak{K}\left(\mathcal{N}_{2}^{6}(c ;(0, \varepsilon))\right) \approx \mathfrak{K}\left(\mathcal{N}_{3}^{6}(0, \varepsilon)\right) \approx \mathfrak{K}\left(\mathcal{N}_{4}^{6}(0, \varepsilon)\right)$ $\approx \mathfrak{K}\left(\mathcal{N}_{6}^{6}(0, \varepsilon)\right) \approx \mathfrak{K}\left(\mathcal{N}_{1}^{4}\left(c ;\left(0, T^{2}\right)\right)\right) \approx \mathfrak{K}\left(\mathcal{N}_{2}^{4}\left(\kappa, \theta ;\left(0, T^{2}\right)\right)\right)$.

3. $A_{4,9}^{0} \approx \mathfrak{K}\left(\mathcal{N}_{5}^{6}(0, \varepsilon)\right) \approx \mathfrak{K}\left(\mathcal{N}_{3}^{4}\left(c ;\left(0, T^{2}\right)\right)\right)$.

4. $\mathfrak{s o}(2,1) \approx \mathfrak{K}\left(\mathcal{N}_{2}^{6}\left(-\frac{1}{2} ;(\varepsilon, 0)\right)\right) \approx \mathfrak{K}\left(\mathcal{N}_{1}^{3}\left( \pm ;\left(T^{1}, 0\right)\right)\right) \approx \mathfrak{K}\left(\mathcal{N}_{2}^{3}\left(c ;\left(T^{1}, 0\right)\right)\right)$ $\approx \mathfrak{K}\left(\mathcal{N}_{3}^{3}(0,0)\right) \approx \mathfrak{K}\left(\mathcal{N}_{4}^{3}(0,0)\right)$.

Note that for the particularly interesting cases $\mathcal{N}_{3}^{3}$ and $\mathcal{N}_{4}^{3}$ (the Lorentzian and Riemannian hyperbolic planes) any torsion perturbation reduces their Lie algebra of affine Killing vectors 
from $\mathfrak{s o}(2,1)$ to $\mathfrak{K}_{\mathcal{B}}$. These two surfaces, together with $\mathcal{N}_{1}^{6}( \pm)$, are the only cases of homogeneous Type $\mathcal{B}$ surfaces which under any perturbation with a torsion tensor reduces the Lie algebra of affine Killing vectors to $\mathfrak{K}_{\mathcal{B}}$.

\section{Conclusions}

Possible extensions of general relativity are based on the independence between the metric and the affine properties of spacetime $[2,3,33]$. In this context torsion plays a fundamental role. In the present article we examine the effects of torsion on the affine Killing vectors of a surface. Since we consider homogeneous surfaces we have a large number of symmetries that preserve the affine connection. In fact, even flat surfaces with non-zero torsion tensor have a very rich structure.

In this paper we have obtained a complete description of the Lie algebra $\mathfrak{K}(\mathcal{M})$ of affine Killing vectors fields on any homogeneous surface $\mathcal{M}$ with non-vanishing torsion. In the Type $\mathcal{A}$ setting $\mathfrak{K}(\mathcal{M})$ is restricted to be one of the following: $\mathfrak{K}_{\mathcal{B}} \oplus \mathfrak{K}_{\mathcal{B}}, A_{4,9}^{0}, A_{12}^{4}$, or $\mathfrak{K}_{\mathcal{A}}$. In the Type $\mathcal{B}$ setting, $\mathfrak{K}(\mathcal{M})$ can only be one of the following: $\mathfrak{K}_{\mathcal{B}} \oplus \mathfrak{K}_{\mathcal{B}}, A_{4,9}^{0}, \mathfrak{s o}(2,1)$, or $\mathfrak{K}_{\mathcal{B}}$. This completes the analysis of [31].

We believe that a systematic description of affine structures with torsion is useful in the search of interesting non-metrizable geometries. A detailed classification of homogeneous surfaces in terms of the torsion tensors they admit is currently in progress. There is no immediate extension of this work to the higher-dimensional setting since there is no analogous classification of the possible affine models, even if torsion is absent. However, we recall that lemma 1.4 holds in any dimension; it is plausible that the analysis of the addition of torsion to a given torsion-free connection at the level of the affine Killing equations could give some insight on possible approaches to the problem.

\section{Acknowledgments}

Research of DD was partially supported by Universidad Nacional de La Plata under Grant 874/18 and Project 11/X791. Research of PBG was partially supported by Project MTM201675897-P (AEI/FEDER, Spain). Research of PP was partially supported by a FulbrightCONICET scholarship and by Universidad Nacional de La Plata under Project 11/X615. DD and PP thank the warm hospitality at the Mathematics Department of the University of Oregon, where this work was carried out.

\section{ORCID iDs}

P Pisani (ㄷ) https://orcid.org/0000-0002-3510-8527

\section{References}

[1] Zanelli J 2005 Lecture Notes on Chern-Simons (Super-)Gravities 2nd edn (arXiv:hep-th/0502193 [hep-th])

[2] Hehl F W, McCrea J D, Mielke E W and Ne'eman Y 1995 Metric affine gauge theory of gravity: field equations, Noether identities, world spinors, and breaking of dilation invariance Phys. Rep. 2581 
[3] Iosifidis D 2019 Exactly solvable connections in metric-affine gravity Class. Quantum Grav. 36085001

[4] Klemm D and Ravera L 2018 Einstein manifolds with torsion and nonmetricity (arXiv:1811.11458 [gr-qc])

[5] Trautman A 1973 Spin and torsion may avert gravitational singularities Nature 2427

[6] Popławski N 2016 Universe in a black hole in Einstein-Cartan gravity Astrophys. J. 83296

[7] Shapiro I L 2002 Physical aspects of the space-time torsion Phys. Rep. 357113

[8] Blagojevic M and Cvetkovic B 2014 Siklos waves with torsion in 3D J. High Energy Phys. JHEP11(2014)141

[9] Babourova O V, Frolov B N, Khetseva M S and Markova N V 2018 Structure of plane gravitational waves of nonmetricity in affine-metric space Class. Quantum Grav. 35175011

[10] Babourova O V, Frolov B N and Klimova E A 1999 Plane torsion waves in quadratic gravitational theories Class. Quantum Grav. 161149

[11] Yu Baurov A, Pronin P I and Stepanyantz K V 2018 Quantum properties of affine-metric gravity with the cosmological term Class. Quantum Grav. 35085006

[12] Hammond R T 2002 Torsion gravity Rep. Prog. Phys. 65599

[13] Bilen L and Gezer A 2018 On metric connections with torsion on the cotangent bundle with modified Riemannian extension J. Geom. 1096

[14] Branding V and Kröncke K 2017 The Ricci flow with metric torsion on closed surfaces J. Geom. Anal. 27 2098-117

[15] Gezer A and Magden A 2017 Geometry of the second-order tangent bundles of Riemannian manifolds Chin. Ann. Math. B 38 985-98

[16] Kupferman R and Maor C 2016 Riemannian surfaces with torsion as homogenization limits of locally Euclidean surfaces with dislocation-type singularities Proc. R. Soc. Edinburgh 146A 741-68

[17] Mozhey N P 2018 Connections of nonzero curvature on homogeneous spaces of unsolvable transformations groups Sib. Èlektron. Mat. Izv. 15 773-85

[18] Yu C 2017 Curvature identities on almost Hermitian manifolds and applications Sci. China Math. 60 285-300

[19] Arias-Marco T and Kowalski O 2008 Classification of locally homogeneous affine connections with arbitrary torsion on 2-manifolds Mh. Math. 1531

[20] Opozda B 2004 A classification of locally homogeneous connections on 2-dimensional manifolds Differ. Geo. Appl. 21 173-98

[21] Grumiller D, Kummer W and Vassilevich D V 2002 Dilaton gravity in two-dimensions Phys. Rep. 369327

[22] Obukhov Y N and Hehl F W 1998 Black holes in two-dimensions Lect. Notes Phys. 514 289-316

[23] Katanaev M O and Volovich I V 1990 Two-dimensional gravity with dynamical torsion and strings Ann. Phys. 1971

[24] Hall G S 1989 The global extension of local symmetries in general relativity Class. Quantum Grav. 6 157-61

[25] Nomizu K 1960 On local and global existence of Killing vector fields Ann. Math. 72 105-20

[26] Bilen L and Gezer A 2017 Some results on Riemannian g-natural metrics generated by classical lifts on the tangent bundle Eurasian Math. J. 8 18-34

[27] Vanžurová A 2016 On metrizable locally homogeneous connections in dimension two Acta Univ. Palack. Olomuc. Fac. Rerum Nat. Math. 55 157-66

[28] Kobayashi S and Nomizu K 1996 Foundations of Differential Geometry vols I and II (New York: Wiley)

[29] Patera J, Sharp R T, Winternitz P and Zassenhaus H 1976 Invariants of real low dimension Lie algebras J. Math. Phys. 17986

[30] Brozos-Vázquez M, García-Río E and Gilkey P 2019 On Distinguished local coordinates for locally homogeneous affine surfaces (arXiv:1901.03523 [math.DG])

[31] Brozos-Vázquez M, García-Río E and Gilkey P 2018 Homogeneous affine surfaces: affine Killing vector fields and gradient Ricci solitons J. Math. Soc. Japan $7025-70$

[32] Gilkey P and Valle-Regueiro X 2019 Applications of PDEs to the study of affine surface geometry Mat. Vesnik 71 45-62

[33] Beltrán Jiménez J, Heisenberg L and Koivisto T S 2019 The geometrical trinity of gravity (arXiv:1903.06830 [hep-th]) 